\title{
Numerical Approaches for Loads and Motions Assessment of Floating WECs Moored by Means of Catenary Mooring Systems
}

\author{
Imanol Touzon ${ }^{10000-0001-9587-4592]}$, Victor Petuya ${ }^{2}$ [0000-0002-4120-3838], Vincenzo Nava ${ }^{1,3}$ \\ ${ }^{[0000-0002-3207-3517]}$, Maria Alonso-Reig ${ }^{1[0000-0003-4999-5937]}$, Iñigo Mendikoa ${ }^{10000-0003-0346-}$ \\ $4161]$ \\ ${ }^{1}$ Tecnalia, Basque Research and Technology Alliance (BRTA), Edificio 700, 48160, Derio, \\ Bizkaia, Spain \\ ${ }^{2}$ Department of Mechanical Engineering, University of the Basque Country - UPV/EHU, Spain \\ ${ }^{3}$ Basque Centre for Applied Mathematics, BCAM, Bilbao, Spain \\ imanol.touzon@tecnalia.com
}

\begin{abstract}
Technologies for harvesting offshore renewable energy based on floating platforms, such as offshore wind, wave and tidal energies, are currently being developed with the purpose of achieving a competitive cost of energy. The economic impact of the mooring system is significant within the total cost of such deployments, and large efforts are being carried out to optimize designs. Analysis of mooring systems at early stages generally require a trade-off between quick analysis methods and accuracy to carry out multi-variate sensitivity analyses. Even though the most accurate approaches are based on the non-linear finite element method in the time domain, these can result in being very time consuming. The most widely used numerical approaches for mooring line load estimates are introduced and discussed in this paper. It is verified that accurate line tension estimates require lines drag and inertia forces to be accounted for. A mooring and floating structure coupled model based on the lumped mass finite element approach is also discussed, and it is confirmed that the differences found in the coupled numerical model are mainly produced by the uncertainty on hydrodynamic force estimates on the floating structure rather than by the lumped mass method. In order to enable quick line tension estimates, a linearization of the structure and mooring coupled model is discussed. It shows accurate results in operational conditions and enables modal analysis of the coupled system.
\end{abstract}

Keywords: Affordable and Clean Energy (SDG7), Multibody Dynamics, Catenary Mooring, Offshore Renewable Energy, Oscillating Water Column. 


\section{Introduction}

Offshore renewable energies have already become relevant in the energy generation mix, especially bottom-fixed offshore wind, that has been commercially deployed for years. In addition, technologies based on floating structures for harvesting wind, tidal currents and wave energy, can be deployed in a wider variety of locations. They represent the largest untapped potential and exploiting a reasonable part of it aids in developing affordable and clean energy (SDG7).

Wave Energy Conversion technologies are being developed ${ }^{1-3}$ with the purpose to be deployed in groups of several devices and the cost of the mooring system is a relevant item within the total array cost ${ }^{4}$. Therefore, it is very interesting that the estimates of the mooring cost are monitored along the path to commercialization, well estimated for single device and afterwards extrapolated to arrays of wave energy converters (WECs).

The sizing of mooring systems tends generally to be very influenced by extreme environmental conditions ${ }^{5}$ but are also subject to fatigue loading under operational conditions. Unlike other mechanical systems, mooring lines are generally described by non-linear models and, given the required low natural frequencies to horizontal motions compared to wave frequencies (WF), mooring analysis needs long time domain (TD) simulations to define their statistics. Therefore, a design process in which multi-variate sensitivity analyses are required, can be difficult to be carried out with the most common numerical methods, based on the non-linear finite element method (FEM) solved in the time domain, as introduced by ${ }^{6-9}$. There are alternative methods to reduce the computation time, that consider catenary lines as quasistatic systems, solved both in the frequency ${ }^{10}$ and in the time domains. Several standardization bodies provide guidelines on how to apply such methods for traditional offshore structures based on quasistatic mooring lines ${ }^{11}$. However, wave energy conversion structures are very dynamically excited and further assumptions may be necessary in order to use the mentioned quasistatic methods, as suggested in ${ }^{12}$.

In order to ease the design process at different development stages, several models are discussed, combining floating structure and mooring models. The corresponding numerical approaches are introduced herein to consider floater and mooring lines coupled, of increasing complexity. The coupling scheme introduced herein is based on multibody dynamics, offering a systematic and feasible coupling between mechanical systems.

\section{Numerical Approaches for Floating Offshore Structures}

During the design of any moored floating structure, it is generally recommended, as a first step, to assess the influence of the mooring system on the structure through a single linearized stiffness term and, afterwards, assess mooring line loads imposing the resulting structure motions to a dynamic mooring system ${ }^{13}$. Once suitable designs have been found a fully coupled dynamic analysis is recommended. This process may lead to endless design loops, especially in early stages, when the number of variables is significant 
and the initial levelized cost of energy ( $L C O E$ ) estimates of the whole system is to be reduced as much as possible.

In order to ease the design process at different development stages, the most relevant models are discussed, combining floating structure and mooring numerical models.

All the introduced approaches consider the floater as a dynamic mechanical system, whilst the main difference lies in considering the mooring system as a static or a dynamic system.

\subsection{Quasistatic Linearized Frequency Domain}

It consists in modelling mooring lines as a static mechanical system and the structure as a dynamic one, which is solved in the frequency domain $(Q S F D)$. It is introduced in ${ }^{11}$ for traditional offshore structures, provided it is demonstrated that effects from mooring line dynamics are negligible. The catenary properties are computed based on the catenary equations, as specified in ${ }^{10}$ and described in Fig. 1. The horizontal restoring force of the mooring system is linearized at the estimated mean horizontal offset, based on steady mean forces. The obtained horizontal stiffness is included in surge/sway motion and the equation of motion (1), accounting for the mooring horizontal stiffness, is solved in the frequency domain. Since drag forces are also considered, the system is solved iteratively through either harmonic or statistical linearization, for regular or irregular waves respectively. The maximum obtained motion, processed assuming specific statistical distributions, is added to the mean value so that the design offset is obtained, as shown in Fig. 1.

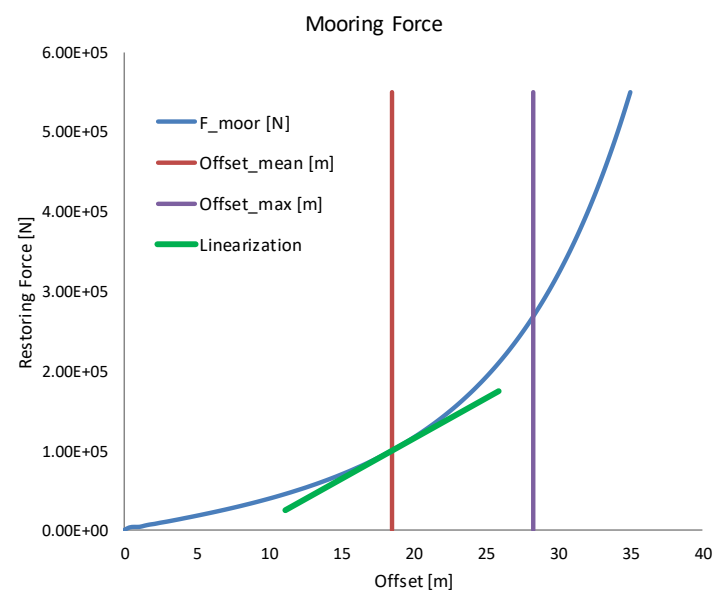

Fig. 1. Mooring static restoring force (blue) and the corresponding linearization (green) at the mean horizontal offset (red), along with the computed maximum offset (purple)

$$
\begin{gathered}
-\omega^{2}\left(\boldsymbol{M}+\boldsymbol{A}(\omega)+\boldsymbol{M}_{\boldsymbol{d k i n}}\right)+i \omega\left(\boldsymbol{B}(\omega)+\boldsymbol{B}_{\boldsymbol{d}}\left(\widehat{\boldsymbol{\delta}}_{a}(\omega)\right)+\boldsymbol{C}_{d k i n}+\boldsymbol{C}_{p t o}\right) \\
+\left(\boldsymbol{K}_{\boldsymbol{h}}+\boldsymbol{K}_{m}+\boldsymbol{K}_{d k i n}\right) \cdot \widehat{\boldsymbol{\delta}}_{\boldsymbol{a}}(\omega)=\hat{\boldsymbol{f}}_{w}(\omega) \cdot \eta_{a}(\omega)
\end{gathered}
$$


Where all bold variables represent vectors and matrices, indicated through lowerand uppercase respectively. $\boldsymbol{M}$ is the body mass matrix, $\boldsymbol{K}_{\boldsymbol{m}}$ and $\boldsymbol{K}_{\boldsymbol{h}}$ indicate linearized mooring and hydrostatic stiffness and $\boldsymbol{A}(\omega)$ and $\boldsymbol{B}(\omega)$ denote the added mass and radiation damping coefficients. Wave excitation force is represented through $\boldsymbol{f}_{w}(\omega)$, which accounts for Froude-Krylov and Diffraction and a linearized drag force is introduced through $\mathbf{B}_{\mathbf{d}}\left(\hat{\delta}_{\mathbf{a}}(\omega)\right)$. $\mathbf{M}_{\mathbf{d k i n}}, \mathbf{C}_{\mathbf{d k i n}}, \mathbf{K}_{\mathbf{d k i n}}$ indicate mass, damping and stiffness matrices from imposing kinematic relations between diffracting bodies, applying (2) with linear restrictions. The resulting motions are represented through the complex amplitude $\widehat{\boldsymbol{\delta}}(\omega)$.

Equation (1) is introduced assuming the modelled floating WEC is made up of several diffracting bodies. In order to build up the corresponding numerical model mentioned bodies move rigidly in specific degrees of freedom whilst the power can be extracted from the relative motions of others. A floating spar type oscillating water column can be modelled trough two diffracting bodies ${ }^{12}$, one representing the floating structure and one representing the surface water level of the internal water column. Therefore, restriction forces ${ }^{14}$ arise in order to impose surge-sway-yaw motions to be rigidly joined, as per equation (2) that, as long as linear relations are set, can be reduced to the set of $\mathbf{M}_{\mathbf{d k i n}}, \mathbf{C}_{\mathbf{d k i n}}$ and $\mathbf{K}_{\mathbf{d k i n}}$ matrices. In addition, the power is extracted with the power take off mechanism, i.e. an air turbine, which is commonly modelled through a damping term, $\mathrm{C}_{\text {pto }}$.

$$
\left(\boldsymbol{M}+\alpha \cdot \boldsymbol{\Phi}_{\delta}{ }^{t} \cdot \boldsymbol{\Phi}_{\delta}\right) \cdot \ddot{\boldsymbol{\delta}}(t)+\alpha \cdot \boldsymbol{\Phi}_{\delta}{ }^{t} \cdot\left(\dot{\boldsymbol{\Phi}}_{\boldsymbol{\delta}} \cdot \dot{\boldsymbol{\delta}}(t)+2 \xi \omega \boldsymbol{\Phi}_{\delta} \cdot \dot{\boldsymbol{\delta}}(t)+\omega^{2} \cdot \boldsymbol{\Phi}(t)\right)=\boldsymbol{f}(t)
$$

The resulting system is solved separately for wave frequency and low frequency motions. First order $W F$ excitation forces are computed with wave amplitudes derived from the spectrum of the corresponding sea state $\left(S_{\eta}(\omega)\right)$. However, the low frequency forces $(L F)$ are computed through the corresponding force amplitude of the slowly varying wave drift force spectrum, showed in equation (3), in the frequency domain, as introduced by Pinkster ${ }^{15}$.

$$
S_{S V}(\mu)=8 \cdot \int_{0}^{\infty} S_{\tau}(\omega+\mu) \cdot S_{\tau}(\omega) \cdot|\boldsymbol{T}(\omega+\mu, \omega)|^{2} \cdot d \omega
$$

Where $S_{S V}(\mu)$ is the slowly varying wave drift force spectrum and $\boldsymbol{T}(\omega, \omega)$ the drift force quadratic transfer function.

In order to ease the computation of the static properties of the mooring system on the floating structure, each mooring configuration, namely number of lines and radial distribution, can be characterized in advance, independently of the floating structure $^{10,12}$.

\subsection{Quasistatic Non-linear Time Domain}

Solving the Cummins equation ${ }^{16}$, showed in (3), accounting for all DOFs in the 3 dimensional space, coupled with the catenary mooring force $\mathbf{f}_{\mathrm{m}}(\mathrm{t})$ is proposed in ${ }^{17}$, and has been assessed in many works, such as ${ }^{7,8}$. It assumes the mooring system as a static mechanical system and the floating structure as a dynamic one, which is solved in the time domain $(Q S T D)$. However, the static (non-linear) properties can be included not 
only in surge but also in all other degrees of freedom. In addition, since the proposed model is solved in the time domain, a nonlinear viscous drag force vector $f_{d}(t)$ in all degrees of freedom can be included, as showed in the last term of the right hand side of (3). The convolution term for the radiation damping can be solved through several methods such as the prony approximation ${ }^{18}$, frequency domain identification ${ }^{19}$ or direct integration, as carried out in ${ }^{9}$. This model is advantageous since it considers the quadratic drag force as well as non-linear geometric stiffness of the mooring system and the influence of all degrees of freedom on mooring lines. However, it requires the catenary equations to be solved at every time step with its implicit iterative loop. The term $\mathbf{f}_{d}(t)$ in (4) represents the viscous drag force vector on the structure.

$$
\begin{aligned}
\left(\boldsymbol{M}+\boldsymbol{A}_{\infty}+\boldsymbol{M}_{\boldsymbol{d k i n}}\right) \cdot \ddot{\boldsymbol{\delta}}(t)+\int_{-\infty}^{t} \boldsymbol{B}(t-\tau) \cdot \dot{\boldsymbol{\delta}}(\tau) \cdot d \tau+\left(\boldsymbol{C}_{\boldsymbol{d k i n}}+\boldsymbol{C}_{\boldsymbol{p t o}}\right) \cdot \dot{\boldsymbol{\delta}}(t) \\
+\left(\boldsymbol{K}_{\boldsymbol{d k i n}}+\boldsymbol{K}_{\boldsymbol{h}}\right) \cdot \boldsymbol{\delta}(t)=\boldsymbol{f}_{\boldsymbol{w}}(t)+\boldsymbol{f}_{\boldsymbol{m}}(t)+\boldsymbol{f}_{\boldsymbol{d}}(t)
\end{aligned}
$$

\subsection{Dynamic Non-linear Time Domain}

In addition to the non-linear geometric stiffness of catenary lines, the most widely used approach to account for drag and inertia forces on lines is the FEM or lumped mass model coupled with the wave structure interaction model, resulting in a completely dynamic model solved in the time domain (DynTD). This model considers the wave structure interaction model based on the linear potential flow complemented with a Morison force term and dynamic moorings based on the lumped mass model. Both can be fully coupled through the use of kinematic relations, as introduced in ${ }^{9}$, so that kinematic restrictions are imposed on all fairleads and anchors of each mooring line. Even though this approach is the most widely used due to its accuracy and availability in commercial codes, it can be too time consuming for sensitivity analyses of designs with several parameters.
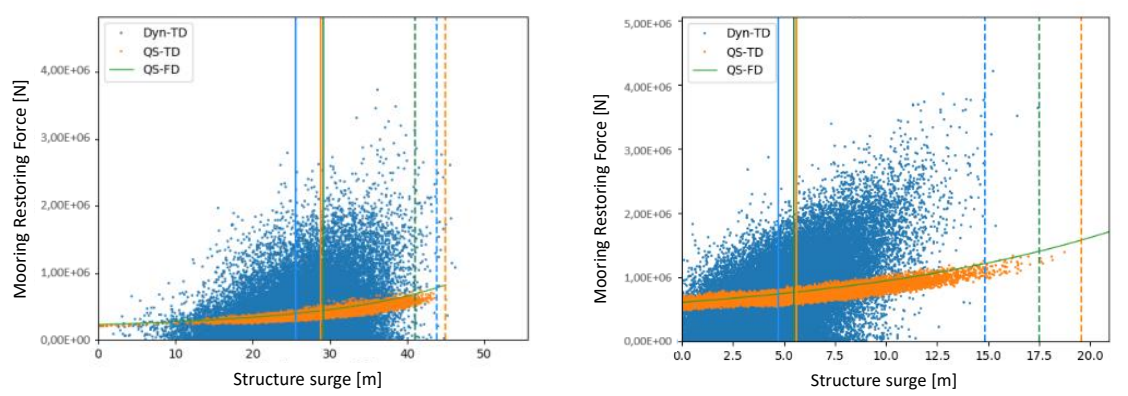

Fig. 2. Line tensions for the corresponding horizontal motions of the structure (surge) with the DynTD (blue), QSTD (orange) and QSFD (green) approaches. Vertical lines represent mean offsets (solid) and design offsets (dashed). Left: low mooring lines pretension; Right: high mooring lines pretension 
Initial results from the case study showed in ${ }^{12}$ and represented in Fig. 2, show that the DynTD approach provides higher uncertainty in terms of the correlation of the restoring force with the horizontal offset of the structure. However, given that DynTD approach accounts for lines drag and inertia forces, this model is considered the most accurate to compute line tensions. When compared with the $Q S F D$ approach, the design line tensions obtained with the DynTD model are two times higher with high line pretensions increasing up to seven times higher with low line pretensions, mostly due to increasing snap load events. Design offsets with low pretensions can be slightly larger with both time domain models due to the influence of the geometric stiffness, which can be covered in the $Q S F D$ approach through appropriate correction factors, see ${ }^{12}$. Nevertheless, with high pretensions, mooring induced damping becomes significant reducing the corresponding design offset, as shown in Fig. 2 right, which may require the use of mooring dynamic approaches in all stages of design.

Therefore, in order to obtain accurate line tensions and representation of the mooring induced damping with high pretensions, it is worth considering the non-linear DynTD approach. The resulting hydrodynamic and mooring coupled model can be summarized through a set of mass, damping and stiffness matrices together with a force vector as in equation (5):

$$
\begin{aligned}
{\left[\begin{array}{cc}
(\boldsymbol{M}+\boldsymbol{A})_{\text {str }}+\boldsymbol{M}_{\boldsymbol{d k i n}} & \boldsymbol{M}_{\boldsymbol{f} / \boldsymbol{a}} \\
\boldsymbol{M}_{\boldsymbol{f} / \boldsymbol{a}} & \boldsymbol{M}_{\text {moor }}
\end{array}\right] \cdot\left\{\begin{array}{c}
\ddot{\boldsymbol{\delta}}_{\text {str }}(t) \\
\ddot{\boldsymbol{\delta}}_{\text {moor }}(t)
\end{array}\right\}+\left[\begin{array}{cc}
\boldsymbol{C}_{\boldsymbol{p t o}}+\boldsymbol{C}_{\boldsymbol{d k i n}} & \boldsymbol{C}_{\boldsymbol{f} / \boldsymbol{a}} \\
\boldsymbol{C}_{\boldsymbol{f} / \boldsymbol{a}} & \boldsymbol{C}_{\text {moor }}
\end{array}\right] \cdot\left\{\begin{array}{c}
\dot{\boldsymbol{\delta}}_{\text {str }}(t) \\
\dot{\boldsymbol{\delta}}_{\text {moor }}(t)
\end{array}\right\} } \\
+\left[\begin{array}{cc}
\boldsymbol{H}_{\text {str }}+\boldsymbol{K}_{\boldsymbol{p t o}}+\boldsymbol{K}_{\boldsymbol{d k i n}} & \boldsymbol{K}_{\boldsymbol{f} / \boldsymbol{a}} \\
\boldsymbol{K}_{\boldsymbol{f} / \boldsymbol{a}} & \boldsymbol{K}_{\text {moor }}
\end{array}\right] \cdot\left\{\begin{array}{c}
\boldsymbol{\delta}_{\text {str }}(t) \\
\boldsymbol{\delta}_{\text {moor }}(t)
\end{array}\right\} \\
=\left\{\begin{array}{c}
\boldsymbol{f}_{\boldsymbol{f} \boldsymbol{k}}(t)+\boldsymbol{f}^{s v}(t)+\boldsymbol{f}_{\boldsymbol{d}}(t)-\boldsymbol{f}_{\text {conv }}(t)-\boldsymbol{f}_{\boldsymbol{f} / \boldsymbol{a}}(t) \\
\boldsymbol{f}_{\boldsymbol{z}}(t)+\boldsymbol{f}_{\boldsymbol{f}}(t)+\boldsymbol{f}_{\boldsymbol{g}}+\boldsymbol{f}_{\boldsymbol{b}}+\boldsymbol{f}_{\text {morison }}(t)+\boldsymbol{f}_{\boldsymbol{f} / \boldsymbol{a}}(t)
\end{array}\right\}
\end{aligned}
$$

Subscripts str denotes structure, moor denotes mooring, pto denotes power take off and $d$ kin and f/a denotes kinematic relations between diffracting bodies and fairleads/anchors respectively. In the force vector referred to the structure, a quadratic viscous drag term $\boldsymbol{f}_{d}(t)$ has been added, considering usual viscous drag coefficients as provided in offshore standards ${ }^{20}$. Due to the differences in natural frequencies of the floating structure and lines' elements, equation (5) represents a stiff system and implicit integration schemes are recommended such as the generalized-alpha methods or Newmark method ${ }^{21}$. It should be noted that the force $\boldsymbol{f}_{f / a}$ stands for the forces to maintain fairleads at a constant distance from the center of gravity of the structure and anchors fixed in the corresponding seabed positions.

The dynamic system described above is composed of three main parts, the floater, mooring lines and the lines fairleads and anchor. All of them have been included in the numerical model either through stiffness, damping and mass matrices or as time varying forces. Floater motions are linear since its wave structure interaction have been computed with a linear potential code and, hence, its matrices are time invariant as well as the power take off (PTO) forces, that are usually modelled through a set of linear stiffness and damping matrices.

On the other hand, kinematics of fairlead and anchor points have been defined by means of multibody restrictions. These restrictions are shown in equations (6). 
$\boldsymbol{\Phi}_{\text {fair }}=\left\{\begin{array}{c}x_{\text {surge }}+x_{\text {roll }}+x_{\text {yaw }}+\delta_{x-\text { fair }}-x_{n} \\ y_{\text {sway }}+y_{\text {pitch }}+y_{\text {yaw }}+\delta_{y-\text { fair }}-y_{n} \\ z_{\text {yaw }}+z_{\text {roll }}+z_{\text {pitch }}+\delta_{z-\text { fair }}-z_{n}\end{array}\right\} \boldsymbol{\Phi}_{\text {anchor }}=\left\{\begin{array}{l}\delta_{x-\text { anchor }}-x_{l} \\ \delta_{y-\text { anchor }}-y_{1} \\ \delta_{z-\text { anchor }}-z_{l}\end{array}\right\}$
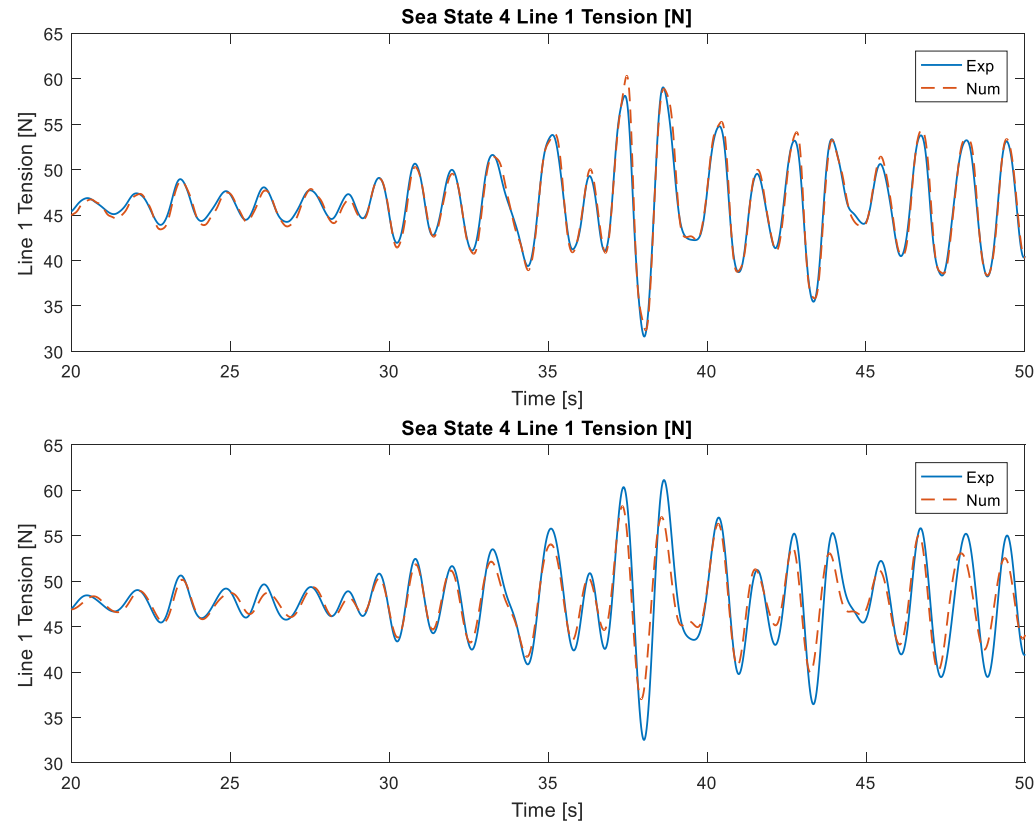

Fig. 3. Line tensions obtained with numerical model compared with the corresponding experimental results. Imposed motions on the fairlead (top) and fully coupled simulation (bottom)

In equation (6) $\delta_{x, y, z,- \text { fair }}$ indicates the position of the fairlead with respect to the center of gravity $(C O G)$ of the structure as well as $x_{\text {surge,roll,yaw }}, y_{\text {sway,pitch,yaw }}$ and $z_{\text {heave, roll,pitch }}$ denote the motions of the fairleads in the global $\mathrm{X}, \mathrm{Y}$ and $\mathrm{Z}$ axis due to the corresponding motions of the structure. The anchor points are to be kept fixed on the seabed and to do so the position with respect to the $C O G$ of the structure $\delta_{x, y, z-a n c h o r}$ are to be updated along the time.

Comparison between experimental and numerical results is introduced in Fig. 3. It represents and Catenary Anchor Leg Moored (CALM) buoy, moored by means of three catenary mooring lines (Lines 1 and 2 windward lines and Line 3 the leeward line). The experimental tests were carried out at a 1:13.6 scale obtaining good agreement between the numerical and experimental results. An in depth analysis of the comparison between experimental and numerical results of the DynTD approach has been introduced in ${ }^{9}$. The comparison is made in two main steps, imposed motions on the numerical model computing the corresponding line tensions, and simulating the fully coupled model of the floating structure and mooring system. It shows that the non-linear lumped mass method provides very accurate results when the motions of the floater are imposed. However, if the coupled system is solved, the accuracy of line tension estimates is reduced. It is attributed to not having modelled non-linearities of the floating structure. 


\subsection{Dynamic Linearized Frequency Domain}

Considering mooring lines as dynamic mechanical systems, coupled to a dynamic floater in a model that can be solved in the frequency domain (DynFD), can be found just in a few references, such as in ${ }^{22}$ applied in ultra-deep waters. It consists in solving the coupled system (5) in the frequency domain $(F D)$, and in order to do so all forces arising from both the structure, the mooring system and line attachments need to be linearized. Main sources of non-linearity are the viscous forces on both the floater and mooring nodes, the geometric stiffness of catenary lines and the fairlead and anchor relations between the structure and mooring line ends. These effects are linearized in this approach in order to enable the $F D$ solution of the whole coupled model, as specified in ${ }^{23}$, as well as the modal analysis, represented in Fig. 4.

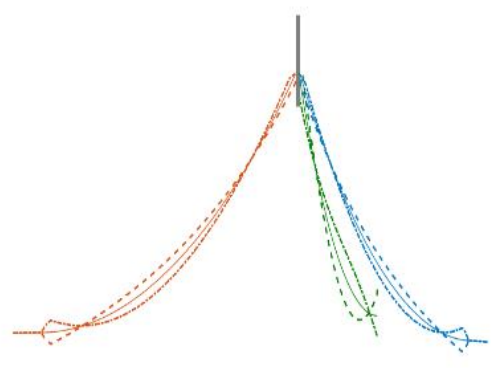

Fig. 4. Mode of the floating structure coupled with the mooring system, computed through modal analysis based in the DynFD approach

The linearization of the Morison viscous drag term ends up in a set of two linearized forces, proportional to the fluid and to the corresponding $D O F$ velocities respectively. Consequently, the damping matrix and the velocity force depend on all DOF motions, implying the $F D$ solution to be solved through a fixed-point iterative process, resulting in harmonic or statistical linearization process for regular or irregular waves respectively.

In contrast with the DynTD model, the DynFD model provides only the time-varying part of the solution and structural and stiffness matrix should be reviewed to work with the time varying part of the solution of motions.

The non-linear geometric stiffness contributes significantly to the system performance, especially in cases with significant mooring pretensions and in the $L F$ range. Its influence on both the floater and mooring lines can be obtained through the secant stiffness force on the structure, as carried out in ${ }^{23}$ accounting for two standard deviations of each degree of freedom of the floater about the mean position.

Kinematic relations, modeling fairlead and anchor points, are defined following equation (2) by means of Lagrange multipliers. Such relations can be broken down into two terms, those depending on model motions and constant forces. Constant forces are 
not included in the frequency domain solution since it is already in equilibrium at the mean position.

The slowly varying second order wave drift forces are included in the model through the spectrum defined in equation (3) and proposed in ${ }^{15}$. Seabed vertical reaction is modelled in the frequency domain through stiffness and damping matrices on the nodes on the seabed in the equilibrium position and the friction force has also been modelled through a damping matrix, applying the same damping coefficient as in the vertical motions.

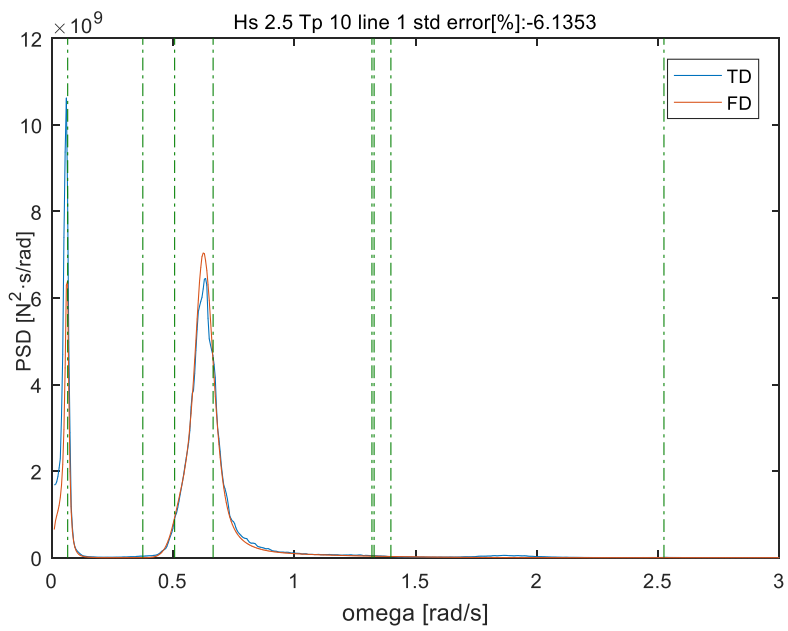

Fig. 5. Comparison of line tensions of the linearized frequency domain (red) with the non-linear time domain (blue) fully coupled models

Linearization of the coupled system provides very good results of both body motions and line tensions when compared with the corresponding non-linear time domain model, see Fig. 5. Accuracy is partially lost in surge and line tension estimates in very low and very high energy sea states, due to the influence of a coupled mode of motion and uncertainties in the mooring induced motion respectively.

\section{Discussion}

It is been verified that preliminary cost estimates, based on extreme environmental conditions, can be obtained with the $Q S F D$ approach for mid to low pretensions, provided total suspended lines length and lines tension are corrected with correction factors. In addition to the non-linear geometric stiffness, it is showed that drag and inertia effects are of utmost importance for line tension estimates. The non-linear lumped mass modelling approach provides very good results when the motion of the fairlead is imposed. Larger uncertainties are introduced in the fully coupled model due to non-linearities of floater body motions. Finally, since the mentioned DynTD model resolves the mooring system and floater motions numerical models in a fully coupled single mechanical system, it has been linearized, yielding the DynFD approach. Such a model is resolved in 
the frequency domain, coupling structure and line motions and accounting for lines drag and inertia as well as the linearized geometric stiffness on catenary mooring lines. The discussed DynFD model has been verified to provide good agreement in operational sea states.

\section{Conclusions}

In this paper a review of numerical modelling methods of catenary-moored floating structures is introduced, with an emphasis on WEC technologies, generally smaller and more dynamically excited than the traditional offshore structures. Summing up, preliminary cost estimates can be obtained with the $Q S F D$ approach for mid to low pretensions. The non-linear lumped mass modelling approach provides itself very good results when the motion of the fairlead is imposed. Larger uncertainties are introduced in floater body motions that are translated into line tension uncertainties. The fully coupled frequency domain approach discussed here has demonstrated good accuracy in operational conditions and it can efficiently be used to assess fatigue damage and control strategies accounting for the mooring influence.

The applicability of the fully coupled frequency domain approach to fatigue life estimates can be very beneficial in terms of computational time. It might, not only enable accounting for the fatigue damage earlier in the design process, but also to be integrated within different kinds of digital twins of floating platforms and its mooring systems. In addition, complex control algorithms of floating renewable energy structures can be implemented with the purpose of minimizing loads on mooring lines. Such control subroutines may require linearized systems of the floating structure and mooring lines to be solved systematically.

These numerically efficient approaches can facilitate further developing offshore renewable energy technologies at early stages, contributing in developing affordable and clean energy (SDG7). Authors are currently working in applying frequency domain approaches to floating offshore wind turbines, also aligned with the SDG7.

\section{Acknowledgments}

This work was funded by Project IT949-16 (Departamento de Educación, Política Lingüística y Cultura, Regional). Government of the Basque Country.

\section{References}

1. MARMOK-A-5 Wave Energy Converter | Tethys. https://tethys.pnnl.gov/project-sites/marmok-5-wave-energy-converter.

2. http://www.corpowerocean.com/technology/. http://www.corpowerocean.com/technology/.

3. Ireland leads the way in ocean energy as SEAI funded wave energy device arrives in Hawaii for testing. Sustainable Energy Authority Of Ireland https://www.seai.ie/news-and-media/ocean-energy-test-device/. 
4. OES | News | International LCOE for Ocean Energy Technology. https://www.ocean-energysystems.org/news/international-lcoe-for-ocean-energy-technology/.

5. Muliawan, M. J., Karimirad, M., Gao, Z. \& Moan, T. Extreme responses of a combined spartype floating wind turbine and floating wave energy converter (STC) system with survival modes. Ocean Eng. 65, 71-82 (2013).

6. H.J.J. Van den Boom. Dynamic Behaviour of Mooring Lines. Behav. Offshore Struct. Elsevier Sci. Publ. B V 359-368 (1985).

7. Azcona, J., Munduate, X., González, L. \& Nygaard, T. A. Experimental validation of a dynamic mooring lines code with tension and motion measurements of a submerged chain. Ocean Eng. 129, 415-427 (2017).

8. Hall, M. \& Goupee, A. Validation of a lumped-mass mooring line model with DeepCwind semisubmersible model test data. Ocean Eng. 104, 590-603 (2015).

9. Touzon, I., Nava, V., Gao, Z., Mendikoa, I. \& Petuya, V. Small scale experimental validation of a numerical model of the HarshLab2.0 floating platform coupled with a non-linear lumped mass catenary mooring system. Ocean Eng. 200, 107036 (2020).

10.Touzon, I. et al. Mooring System Design Approach: A Case Study for MARMOK-A Floating OWC Wave Energy Converter. in (American Society of Mechanical Engineers Digital Collection, 2018). doi:10.1115/OMAE2018-77634.

11.DNVGL-OS-E301: Position mooring. http://rules.dnvgl.com/docs/pdf/dnvgl/os/201807/dnvgl-os-e301.pdf (2018).

12.Touzon, I., Nava, V., de Miguel, B. \& Petuya, V. A Comparison of Numerical Approaches for the Design of Mooring Systems for Wave Energy Converters. J. Mar. Sci. Eng. 8, 523 (2020).

13.Offshore Renewable Energy, Committee v.4. in Proceedings of the 20th International Ship and Offshore Structures Congress (ISSC 2018) (eds. International Ship \& Offshore Structures Congress, Kaminski, M. L., Rigo, P., \& IOS Press) (2018). doi:10.3233/978-1-61499-864-8193.

14.Garcia De Jalon, J. Kinematic and dynamic simulation of multibody systems: the realtime challenge. (Springer, 1994).

15.Pinkster, J. A. \& Basin, N. S. M. Low Frequency Second Order Wave Exciting Forces on Floating Structures. 220.

16.Cummins, W. E. The Impulse Response Function and Ship Motions. in (1962).

17.NI 461 DTO ROO E - Quasi-Dynamic Analysis of Mooring Systems using Ariane Software. (1997).

18.Duclos, G., Clement, A. H. \& Chatry, G. Absorption of Outgoing Waves in a Numerical Wave Tank Using a Self-Adaptive Boundary Condition. Int. J. OFFSHORE POLAR Eng. 11, 168175 (2001).

19.Perez, T. \& Fossen, T. I. Time-domain vs. Frequency-domain Identification of Parametric Radiation Force Models for Marine Structures at Zero Speed. Identification Control 19.

20.DNV-RP-C205: Environmental Conditions and Environmental Loads. 124 (2010).

21.Naess, A. \& Moan, T. Stochastic Dynamics of Marine Structures. (Cambridge: Cambridge University Press, 2012). doi:10.1017/CBO9781139021364.

22.Low, Y. M. \& Langley, R. S. Time and frequency domain coupled analysis of deepwater floating production systems. Appl. Ocean Res. 28, 371-385 (2006).

23.Touzon, I., Nava, V., Gao, Z. \& Petuya, V. Frequency domain modelling of a coupled system of floating structure and mooring Lines: An application to a wave energy converter. Ocean Eng. 220, 108498 (2021). 